\title{
Management of urinary tract infections in patients with neurogenic bladder: challenges and solutions
}

\section{Jürgen Pannek \\ Jens Wöllner}

Neuro-Urology, Swiss Paraplegic Center, Nottwil, Switzerland
Correspondence: Jürgen Pannek Neuro-Urology, Schweizer ParaplegikerZentrum, Guido A Zäch Strasse I, $\mathrm{CH}-6207$ Nottwil, Switzerland

$\mathrm{Tel}+4|4| 9395924$

Fax +4I 419395923

Email juergen.pannek@paraplegie.ch
This article was published in the following Dove Press journal:

Research and Reports in Urology

II July 2017

Number of times this article has been viewed

Introduction: Urinary tract infections (UTIs) are one of the most common morbidities in persons with neurogenic lower urinary tract dysfunction (NLUTD). They are associated with a significant morbidity and mortality, and they affect the quality of life of the affected patients. Diagnosis and treatment of UTI in this group of patients are challenging. In this review, the current strategies regarding diagnosis, treatment, and prevention are summarized.

Diagnostics: it is important to correctly diagnose a UTI, as treatment of bacteriuria should strictly be avoided. A UTI is defined as a combination of laboratory findings (leukocyturia and bacteriuria) and symptoms. Laboratory findings without symptoms are classified as asymptomatic bacteriuria. Routine urine screening is not advised.

Treatment: Only UTI should be treated; treatment of asymptomatic bacteriuria is not indicated. Prior to treatment, urine for a urine culture should be obtained. Antibiotic treatment for $\sim 7$ days is advised.

Prevention: In recurrent UTI, bladder management should be optimized and morphologic causes for UTI should be excluded. If UTIs persist, medical prophylaxis should be considered. Currently, no prophylactic measure with evidence-based efficacy exists. Long-term antibiotic prophylaxis should be used merely as an ultimate measure. Among the various mentioned innovative approaches for UTI prevention, bacteriophages, intravesical instillations, complementary and alternative medicine techniques, and probiotics seem to be most promising.

Conclusion: Recently, several promising innovative options for UTI prophylaxis have been developed which may help overcome the current therapeutic dilemma. However, further well designed studies are necessary to evaluate the safety and efficacy of these approaches.

Keywords: neurogenic lower urinary tract dysfunction, spinal cord injury, prophylaxis, resistance, lactobacilli, cranberries

\section{Introduction}

Urinary tract infections (UTIs) are one of the most common morbidities in individuals with neurogenic lower urinary tract dysfunction (NLUTD). ${ }^{1}$ Approximately one in five individuals with NLTUD suffers from recurrent UTI, which are associated with a considerable morbidity and mortality and affect the quality of life (QoL) substantially. ${ }^{2}$ UTIs are the leading cause for septicemia in patients with spinal cord lesions, and are associated with a significantly increased mortality. ${ }^{3}$ Furthermore, symptomatic UTIs are often bothersome for patients and are therefore related to a decreased health-related QoL. As UTIs are often recurrent and the bacterial strains are increasingly resistant to antibiotic treatment, ${ }^{4}$ UTIs present a relevant clinical challenge for both patients and caregivers. 
The increased risk of UTIs in individuals with NLUTD is due to many different factors. In particular, impaired storage and voiding functions are risk factors for UTI, ${ }^{5}$ either because of the dysfunction itself, for example, detrusor overactivity, ${ }^{6}$ or because of the applied method for bladder management, especially indwelling catheters, and also intermittent catheterization. ${ }^{7}$ Furthermore, morphologic causes, like bladder stones or foreign bodies, ${ }^{5}$ and elevated residual urine ${ }^{8}$ are known sources for UTI.

Thus, the key to controlling UTIs in individuals with NLUTD is to optimize their bladder management and to eliminate sources of infection, such as bladder stones or foreign bodies. Unfortunately, recurrent UTIs persist despite these measures in a high percentage of affected patients. Therefore, several preventive or protective measures have been proposed, but until today, no prophylaxis with evidence-based efficacy exists. ${ }^{9}$ As a consequence, a plethora of different methods is currently used for UTI prophylaxis. In this review, we aim at summarizing the current strategies for UTI treatment and prophylaxis, their limitations, and possible new options.

\section{Definition of UTI}

The definition of a UTI is of utmost importance, as there is broad consensus that only symptomatic UTI should be treated and bacteriuria should not be regarded as an indication for treatment. ${ }^{9}$ Basically, the definition of UTIs in persons with NLUTD does not differ significantly from the definitions used in persons without NLUTD. In accordance with the most recent guidelines, ${ }^{9,10}$ UTIs are defined as the combination of bacteriuria, leukocyturia, and clinical symptoms. Unfortunately, due to the underlying neurologic disorder, symptoms are often not easy to assess, as sensory functions of the lower urinary tract are frequently impaired. Therefore, a data set has been developed by the International Spinal Cord Injury (SCI) Society for defining UTI, ${ }^{10}$ which facilitates the evaluation and documentation of signs and symptoms in this group of patients. For urinalysis, dipstick testing alone has been demonstrated not to be sufficiently sensitive and specific. ${ }^{11}$ Thus, urine culture is mandatory, and leukocyte esterase activity or urine microscopy should be used for the evaluation of leukocyturia. For quantification, no evidence-based cutoff values exist. The most frequently used consensus used $>10^{2}$ colony forming units (cfu) $/ \mathrm{mL}$ as a cutoff for significant bacteriuria if the urine was collected by catheterization, $>10^{4} \mathrm{cfu} / \mathrm{mL}$ for clean void, and any detectable concentration for suprapubic aspirates. ${ }^{9}$ For leukocyturia, often 100 leukocytes $/ \mathrm{mL}$ is regarded as a cutoff value. As only UTIs should be treated, urine screening for asymptomatic bacteriuria is not advised.

\section{UTI treatment}

Numerous guidelines point out that asymptomatic bacteriuria should not be treated. UTI symptoms may be vastly different from those in patients without NLUTD. Often the typical symptoms, for example, dysuria, pain, and frequency are not present. Typical symptoms are increased incontinence, increased spasticity, decreased bladder capacity, malaise, fever, or autonomic dysregulation. If a UTI is diagnosed, there is broad consensus that UTI should be treated with narrow spectrum antibiotics, if possible, for the shortest duration that is clinically safe. ${ }^{12}$ As, by definition, UTIs in persons with NLUTD are considered as complicated UTI, single-shot or short-term treatments (1-3 days) are not advised. Based on the results of a meta-analysis, a 7-10 day treatment for UTI without fever, and 14 days in patients with fever is recommended. ${ }^{12}$ In addition, if the infection involves parenchymal organs (eg, pyelonephritis, prostatitis), the treatment duration should be extended. ${ }^{13}$ In patients with long-term indwelling catheters, the catheter should be changed under treatment. As the bacterial strains causing UTI and the resistance patterns of these bacteria often differ distinctly from those in patients with uncomplicated UTI, treatment should only be started after a urine specimen has been obtained for microbiologic testing. This is necessary to choose the appropriate antibiotic and to avoid bacterial resistance. ${ }^{9,14}$

In patients with uncomplicated cystitis and mild clinical symptoms, nitrofurantoin is a preferred option if sensitive, because of the low resistance rate and marginal alteration of the bowel flora. ${ }^{5}$ In case of severe infection, where an immediate therapy is necessary fluorchinolones are recommended. ${ }^{14}$ Usually, even in patients with febrile UTI, a monotherapy is sufficient. There is no difference in the cure rate comparing treatment with a single substance versus dual therapy ${ }^{15}$ In hospitalized patients with severe infection suspicious for methicillinresistant Staphylococcus aureus (MRSA), vancomycin is an option. In patients with MRSA in an outpatient setting, trimethoprim-sulfamethoxazole enables suitable management. ${ }^{5}$

If UTIs persist despite appropriate treatment, factors that potentially sustain UTI, for example, bladder or kidney stones, elevated post-void residual urine, or abscess formation, have to be identified and treated. ${ }^{16}$

An analysis of the UTI treatment strategies in SCI rehabilitation centers demonstrated that even in specialized units, asymptomatic bacteriuria was treated in $>50 \%$ of the institutions if the amount of bacteria and leukocytes was regarded as significant. Thus, overtreatment seems to exist even in expert centers, with the subsequent risk of selecting multiresistant bacteria. ${ }^{17}$ 


\section{UTI prevention}

Compared to UTI treatment, UTI prevention strategies are less clear-cut, and currently, no evidence-based recommendation exists. ${ }^{9}$ The current lack of successful preventive measures for UTI is owing to the multitude of factors contributing to its pathogenesis. Besides impaired bladder function and morphologic causes, for example, stones, the bladder evacuation method is the main predictor for symptomatic UTIs in individuals with NLUTD, whereas patient and injury characteristics have no significant effect. ${ }^{7,18} \mathrm{An}$ analysis of the data of 1,104 patients with chronic ( $>1$ year) SCI demonstrated that the bladder evacuation method was a significant $(p \leq 0.049)$ predictor for the occurrence of UTIs and recurrent UTIs. The odds of experiencing recurrent UTIs were increased $>5$-fold in patients with transurethral indwelling catheters, and were increased $>10$-fold compared to patients voiding spontaneously. However, also in patients using intermittent catheterization, suprapubic catheterization, or triggered reflex voiding, the odds of a UTI were increased significantly, but less dramatically. ${ }^{7}$ In addition, SCI induces an immune deficiency syndrome that is sufficient to propagate clinical relevant infections in these patients. ${ }^{19}$

Currently, only a minority of the underlying causes can be treated effectively. Therefore, if bladder management has been optimized and morphologic reasons for UTI have been removed, prophylaxis resorts to medical interventions.

\section{Antibiotic prophylaxis}

Chronic bacteriuria and recurrent UTI are common in patients with NLUTD. ${ }^{4}$ Depending on the underlying disease and bladder management, chronic bacteriuria can be unavoidable. It is therefore essential to inform patients and physicians that treatment of asymptomatic bacteriuria is not prudent in these patients, and this information is an important first step in the concept of UTI prophylaxis. Routine antibiotic prophylaxis is not recommended due to the lack of efficacy and the increase in bacterial resistance. ${ }^{12}$ Current literature does not support the routine use of antibiotic prophylaxis in patients with NLUTD due to SCI. ${ }^{20}$ There is no evidence for antibiotic prophylaxis in patients using intermittent catheterization to prevent symptomatic UTI. ${ }^{21}$ A recent meta-analysis demonstrated that antibiotic prophylaxis did not prevent UTI, but doubled the rate of antimicrobial resistance. ${ }^{12}$ Therefore, routine antibiotic prophylaxis is not recommended.

Recently, the concept of weekly oral cycling antibiotics (WOCA) showed promising results. This regimen consisted of the alternate administration of two antibiotics once per week over a period of at least 2 years. There was a significant decrease in the incidence of UTI from 9.4 UTIs per patientyear before intervention to 1.8 UTIs per patient-year. No severe adverse events and no new cases of colonization with multidrug-resistant bacteria were reported..$^{22}$ If these results can be confirmed by other groups, WOCA may be a feasible option for prophylaxis.

\section{Periinterventional prophylaxis for invasive urological procedures}

Patients with NLUTD required regular urodynamic investigations to control lower urinary tract function (eg, videourodynamics). In addition, there is a frequent need for endoscopic interventions (eg, injection of onabotulinum toxin, bladder stone lithotripsy, cystoscopy). Patients with chronic asymptomatic bacteriuria are at risk to develop bacteremia with subsequent septicemia. Unfortunately, data on which recommendations can be based are scarce. In a pilot study, no difference was found between a single pre-procedural dose of antibiotics compared to a 3-5 day course of pre-procedural antibiotics. The authors conclude that a single dose of pre-procedural antibiotics is safe and effective in these patients. ${ }^{23}$ Concerning urodynamic investigations, which are less invasive than surgical interventions, there is an ongoing debate. In the most recent studies, no routine prophylaxis is recommended, ${ }^{24}$ but periinterventional prophylaxis may be required in certain subgroups with additional risk factors, for example, vesico-renal reflux, previous symptomatic UTI after urodynamics, or immunosuppression.

\section{Nonantibiotic prophylaxis}

\section{Phytotherapy}

Cranberry products are extensively used for UTI prophylaxis. In a randomized, double-blind, placebo-controlled study, neither cranberry tablets nor methenamine hippurate was successful in preventing UTI in patients with SCI. ${ }^{25}$ However, the study observation period was only 6 months, which is rather short, and patients with UTI irrespective of the causing bacteria were included. Given the fact that cranberry proanthocyanidins have bacterial anti-adhesion activity on uropathogenic P-fimbriated Escherichia coli, ${ }^{26}$ the efficacy of cranberry products may differ between different bacteria, but currently, no study evaluating this hypothesis is available.

Other drugs used in phytotherapy, for example, a combination of nasturtium and horseradish, or bearberry leave extracts, showed promising results in patients with uncomplicated UTI, but were not systematically assessed in patients with NLUTD. 


\section{Urine acidification}

Concerning urine acidification, evidence for its effectiveness is insufficient. Merely one study with several methodologic problems exists, ${ }^{27}$ and L-methionine, the drug most frequently used for urine acidification, may increase serum homocysteine levels,${ }^{28}$ which is regarded as a risk factor for cardiovascular diseases. ${ }^{29}$

\section{D-Mannose}

D-Mannose is a sugar that is excreted into the urine and binds to bacterial fimbriae. ${ }^{30}$ In a recent study, it was demonstrated that its use is safe also in 22 patients with multiple sclerosis and NLUTD, and initial results regarding its efficacy were promising. ${ }^{31}$ However, further studies are required to determine the usefulness of this promising option.

\section{Probiotics}

Probiotics, especially Lactobacillus reuteri $\mathrm{RC}-14+$ Lactobacillus rhamnosus GR-1, have been demonstrated to be beneficial in preventing uncomplicated UTI in postmenopausal women. Today, it is not known if these substances are effective in preventing UTI in people with SCI, but a large multicenter prospective trial has been designed and is currently ongoing to answer this question. ${ }^{32}$

\section{Immunostimulation}

Oral immunostimulation with OM-89S, a lyophilized lysate of 18 E. coli strains, has been proven effective in uncomplicated UTI. In patients with NLUTD, merely one study exists, showing a statistically significant decrease in the degree of bacteriuria, but a nonsignificant decrease in UTI. ${ }^{33}$

A very recent study evaluated the safety of a new tetravalent $E$. coli bioconjugate vaccine, administered by intramuscular injection. Initial data confirm the safety of the treatment and show an initial trend for less uncomplicated E. coli UTI; further studies are currently being conducted. ${ }^{34}$

\section{Bacterial interference}

The inoculation of nonpathogenic bacteria, mostly E. coli, into the bladder has been tested in several studies with small numbers of participants. Initial results were promising, with sufficient colonization rates of the inoculated pathogenic bacteria and significant reductions in UTI frequency. Currently, however, long-term data and sufficiently powered trials are lacking. ${ }^{35,36}$ In the most recent study, colonization with E. coli HU2117 did not prevent UTI in patients with chronic indwelling catheters. ${ }^{37}$

\section{Bladder irrigation}

The usefulness of bladder irrigation has mainly been studied in patients with indwelling catheters. In a prospective randomized study, neither sterile saline, acetic acid, nor a neomycin-polymyxin solution had an effect on the degree of bacteriuria or pyuria in SCI patients with indwelling catheters. ${ }^{38}$ A Cochrane review came to the conclusion that bladder irrigation has not been proven to be effective for UTI prevention. ${ }^{39}$

\section{Intravesical instillation}

Intravesical instillation of hyaluronic acid and chondroitin sulfate aims at repairing the glycosaminoglycan layer of the bladder, which is a protective means against UTI and is thought to be disrupted by UTI. ${ }^{40}$ Initial data in patients with uncomplicated UTI showed promising results, ${ }^{41}$ but no study in patients with NLUTD exists.

\section{Complementary and alternative medicine (CAM)}

Due to the absence of evidence-based concepts for UTI prevention, patients frequently use CAM therapies. Three out of four chronic SCI patients have used CAM for treatment of their medical problems, mainly pain and UTI. The most frequently used techniques were homeopathy and acupuncture. Patient satisfaction with both techniques regarding UTI prevention was high. ${ }^{42}$

\section{Acupuncture}

Acupuncture is frequently used in SCI patients, but until today, studies evaluating its use in patients with SCI focused on pain or spasticity. We could not find any data concerning UTI prevention in this group. In uncomplicated UTIs, two studies demonstrated that acupuncture resulted in a significant reduction of UTI, ${ }^{43,44}$ but this does not necessarily mean that the procedure is effective in patients with complicated UTI, namely patients with NLUTD, as well.

\section{Homeopathy}

Recently, the usefulness of classical homeopathy as an adjunctive measure for UTI prophylaxis in patients with NLUTD due to SCI has been demonstrated in a case series. ${ }^{45}$ However, prospective studies for evidence-based evaluation of homeopathic treatment are currently not available. Although the exact mode of action needs to be further elucidated, homeopathy is based on stimulating autoregulatory and self-healing processes. ${ }^{46}$ As the high incidence of UTI in patients with SCI seems to be caused not merely by the 
presence of aggressive bacteria, but also by an impaired host defense, the stimulation of these processes may be a possible mode of action of homeopathic prophylaxis.

Patient satisfaction with both forms of CAM treatment mentioned above was high. ${ }^{42}$ Therefore, CAM techniques could be considered in SCI patients with recurrent UTI. At least for homeopathy, evidence is emerging that it is not only well tolerated, but also effective even in patients with complicated UTI.

\section{Bowel management}

Although the exact mechanism of action is not fully understood, optimizing bowel management leads to a significant reduction of UTI in patients with SCI ${ }^{47}$ It is calculated that by improving bowel management, for example, by transanal irrigation, a lifetime $29 \%$ reduction of UTI can be achieved. ${ }^{48}$ Therefore, bowel management should be evaluated in each patient with recurrent UTI.

\section{Bacteriophages}

During the last years, virulent bacteriophages (or phages), which have been used in eastern European countries already in the 1980s, have reemerged for therapeutic purposes. ${ }^{49}$ Phages are viruses that replicate inside the bacterial host. They cause cell lysis and thus subsequently release new phages, which are able to infect neighboring cells. Phages are potential specific antibacterial agents, as they have a self-replicating nature in the presence of host cells, but they are eliminated from the human body in their absence. ${ }^{50}$ Furthermore, they are active against antibiotic-resistant bacteria, and phage preparations containing several phages (phage cocktails) can increase their activity spectrum. ${ }^{51}$

In vitro studies have demonstrated that phages can develop high specific lytic activities for bacteria isolated from patients with UTI due to SCI. In addition, resistance optimization by direct adaption of bacteriophages was possible, ${ }^{52}$ and phages can degrade even uropathogenic $E$. coli biofilms. ${ }^{53}$ Recently, initial case reports of phage use in humans for UTI treatment have been reported. ${ }^{54}$ Therefore, phages may be the most promising future option for treatment and prevention of UTI. Currently, their use is limited due to safety issues, but trials in humans have already been initiated. ${ }^{55}$

\section{Summary}

Treatment of UTI in patients with NLUTD remains a challenge. First of all, it is important to correctly diagnose a UTI, as treatment of bacteriuria should strictly be avoided. Antibiotic treatment is the method of choice today. Prior to treatment, urine should be collected for urine culture. In recurrent UTI, bladder management should be optimized and morphologic causes for UTI should be excluded. Thus, diagnostic evaluation includes urodynamic testing, sonography, imaging of the urinary tract, and endoscopy. It is important not to forget other possible sources of infection. Both bowel management and catheterization technique should be assessed and optimized if necessary. If UTIs persist, long-term antibiotic prophylaxis should be used merely as an ultimate measure in the least number of patients possible. Currently, no prophylactic measure with evidence-based efficacy exists; therefore, prophylaxis remains a trial and error approach. Among the various mentioned innovative approaches for UTI prevention, bacteriophages, intravesical instillations, CAM techniques, and probiotics seem to be the most promising options. However, further well-designed studies are necessary to find adequate solutions for one of the most frequent and most troublesome medical problems in patients with NLUTD.

\section{Disclosure}

The authors report no conflicts of interest in this work.

\section{References}

1. Esclarin De Ruz A, Garcia Leoni E, Herruzo Cabrera R. Epidemiology and risk factors for urinary tract infection in patients with spinal cord injury. J Urol. 2000;164(4):1285-1289.

2. Biering-Sorensen F, Nielans HM, Dorflinger T, Sorensen B. Urological situation five years after spinal cord injury. Scand J Urol Nephrol. 1999;33(3):157-161.

3. Biering-Sørensen F, Bagi P, Høiby N. Urinary tract infections in patients with spinal cord lesions: treatment and prevention. Drugs. 2001;61(9): $1275-1287$

4. Hinkel A, Finke W, Bötel U, Gatermann SG, Pannek J. Increasing resistance against antibiotics in bacteria isolated from the lower urinary tract of an outpatient population of spinal cord injury patients. Urol Int 2004;73(2):143-148.

5. Vigil HR, Hickling DR. Urinary tract infection in the neurogenic bladder. Transl Androl Urol. 2016;5(1):72-87.

6. Game X, Castel-Lacanal E, Bentaleb Y, et al. Botulinum toxin A detrusor injections in patients with neurogenic detrusor overactivity significantly decrease the incidence of symptomatic urinary tract infections. Eur Urol. 2008;53(3):613-618.

7. Krebs J, Wöllner J, Pannek J. Risk factors for symptomatic urinary tract infections in individuals with chronic neurogenic lower urinary tract dysfunction. Spinal Cord. 2016;54(9):682-686.

8. Merritt JL. Residual urine volume: correlate of urinary tract infection in patients with spinal cord injury. Arch Phys Med Rehabil. 1981;62(11) $558-561$.

9. Groen J, Pannek J, Castro Diaz D, et al. Summary of European Association of Urology (EAU) guidelines on neuro-urology. Eur Urol. 2016;69(2):324-333.

10. Goetz LL, Cardenas DD, Kennelly M, et al. International spinal cord injury urinary tract infection basic data set. Spinal Cord 2013;51(9):700-704.

11. Hoffman JM, Wadhwani R, Kelly E, Dixit B, Cardenas DD. Nitrite and leukocyte dipstick testing for urinary tract infection in individuals with spinal cord injury. J Spinal Cord Med. 2004;27(2):128-132. 
12. Everaert K, Lumen N, Kerckhaert W, Willaert P, van Driel M. Urinary tract infections in spinal cord injury: prevention and treatment guidelines. Acta Clin Belg. 2009;64(4):335-340.

13. Pannek J, Pannek-Rademacher S, Cachin-Jus M. Organ-preserving treatment of an epididymal abscess in a patient with spinal cord injury. Spinal Cord. 2014;52 (Suppl 1):S7-S8.

14. Hooton TM, Bradley SF, Cardenas DD, et al. Diagnosis, prevention, and treatment of catheter-associated urinary tract infection in adults: 2009 International Clinical Practice guidelines from the Infectious Diseases Society of America. Clin Infect Dis. 2010;50(5):625-663.

15. Dinh A, Toumi A, Blanc C, et al. Management of febrile urinary tract infection among spinal cord injured patients. BMC Infect Dis. 2016;16(16): 156 .

16. Siroky MB. Pathogenesis of bacteriuria and infection in the spinal cord injured patient. Am J Med. 2002;113 (Suppl 1A):67S-79S.

17. Pannek J. Treatment of urinary tract infection in persons with spinal cord injury: guidelines, evidence, and clinical practice. A questionnairebased survey and review of the literature. J Spinal Cord Med. 2011; 34(1):11-15.

18. Waites KB, Canupp KC, DeVivo MJ. Epidemiology and risk factors for urinary tract infection following spinal cord injury. Arch Phys Med Rehabil. 1993;74(7):691-695.

19. Brommer B, Engel O, Kopp MA, et al. Spinal cord injury-induced immune deficiency syndrome enhances infection susceptibility dependent on lesion level. Brain. 2016;139(Pt 3):692-707.

20. Morton SC, Shekelle PG, Adams JL, et al. Antimicrobial prophylaxis for urinary tract infection in persons with spinal cord dysfunction. Arch Phys Med Rehabil. 2002;83(1):129-138.

21. Niël-Weise BS, van den Broek PJ, da Silva EM, Silva LA. Urinary catheter policies for long-term bladder drainage. Cochrane Database Syst Rev. 2012;8:CD004201.

22. Salomon J, Denys P, Merle C, et al. Prevention of urinary tract infection in spinal cord-injured patients: safety and efficacy of a weekly oral cyclic antibiotic (WOCA) program with a 2 year follow-up - an observational prospective study. J Antimicrob Chemother. 2006;57(4):784-788.

23. Chong JT, Klausner AP, Petrossian A, et al. Pre-procedural antibiotics for endoscopic urological procedures: initial experience in individuals with spinal cord injury and asymptomatic bacteriuria. J Spinal Cord Med. 2015;38(2):187-192.

24. Böthig R, Fiebag K, Thietje R, Faschingbauer M, Hirschfeld S. Morbidity of urinary tract infection after urodynamic examination of hospitalized SCI patients: the impact of bladder management. Spinal Cord. 2013;51(1):70-73.

25. Lee BB, Haran MJ, Hunt LM, et al. Spinal-injured neuropathic bladder antisepsis (SINBA) trial. Spinal Cord. 2007;45(8):542-550.

26. Howell A, Souza D, Roller M, Fromentin E. Comparison of the antiadhesion activity of three different cranberry extracts on uropathogenic P-fimbriated Escherichia coli: a randomized, double-blind, placebo controlled, ex vivo, acute study. Nat Prod Commun. 2015;10(7): 1215-1218.

27. Günther M, Noll F, Nützel R, Gläser E, Kramer G, Stöhrer M. Harnwegsinfektprophylaxe. Urinansäuerung mittels L-Methionin bei neurogener Blasenfunktionsstörung. Urologe B. 2002;42(4):218-220. German.

28. Ditscheid B, Fünfstück R, Busch M, Schubert R, Gerth J, Jahreis G. Effect of L-methionine supplementation on plasma homocysteine and other free amino acids: a placebo-controlled double-blind cross-over study. Eur J Clin Nutr. 2005;59(6):768-775.

29. Stanger O, Herrmann W, Pietrzik K, et al. Consensus paper on the rational clinical use of homocysteine, folic acid and B-vitamins in cardiovascular and thrombotic diseases: guidelines and recommendations. Clin Chem Lab Med. 2003;41(11):1392-1403.

30. Wellens A, Garofalo C, Nguyen H, et al. Intervening with urinary tract infections using anti-adhesives based on the crystal structure of the FimH-oligomannose-3 complex. PLoS One. 2008;3(4):e2040.
31. Phé V, Pakzad M, Haslam C, et al. Open label feasibility study evaluating D-mannose combined with home-based monitoring of suspected urinary tract infections in patients with multiple sclerosis. Neurourol Urodyn. Epub 2016 Nov 4.

32. Lee BB, Toh SL, Ryan S, et al. Probiotics [LGG-BB12 or RC14-GR1] versus placebo as prophylaxis for urinary tract infection in persons with spinal cord injury [ProSCIUTTU]: a study protocol for a randomised controlled trial. BMC Urol. 2016;16:18.

33. Hachen HJ. Oral immunotherapy in paraplegic patients with chronic urinary tract infections: a double-blind, placebo-controlled trial. J Urol. 1990;143(4):759-762.

34. Huttner A, Hatz C, van den Dobbelsteen G, et al. Safety, immunogenicity, and preliminary clinical efficacy of a vaccine against extraintestinal pathogenic Escherichia coli in women with a history of recurrent urinary tract infection: a randomised, single-blind, placebo-controlled phase $1 \mathrm{~b}$ trial. Lancet Infect Dis. 2017;17(5):528-537.

35. Sundén F, Håkansson L, Ljunggren E, Wullt B. Escherichia coli 83972 bacteriuria protects against recurrent lower urinary tract infections in patients with incomplete bladder emptying. J Urol. 2010;184(1):179-185.

36. Darouiche RO, Green BG, Donovan WH, et al. Multicenter randomized controlled trial of bacterial interference for prevention of urinary tract infection in patients with neurogenic bladder. Urology. 2011;78(2):341-346.

37. Horwitz D, McCue T, Mapes AC, et al. Decreased microbiota diversity associated with urinary tract infection in a trial of bacterial interference. J Infect. 2015;71(3):358-367.

38. Waites KB, Canupp KC, Roper JF, Camp SM, Chen Y. Evaluation of 3 methods of bladder irrigation to treat bacteriuria in persons with neurogenic bladder. J Spinal Cord Med. 2006;29(3):217-226.

39. Hagen S, Sinclair L, Cross S. Washout policies in long-term indwelling urinary catheterization in adults. Cochrane Database Syst Rev. 2010;3:CD004012.

40. Iavazzo C, Athanasiou S, Pitsouni E, Falagas M. Hyaluronic acid: an effective alternative treatment of interstitial cystitis, recurrent urinary tract infections, and hemorrhagic cystitis? Eur Urol. 2007;51(6): 1534-1540.

41. Damiano R, Quarto G, Bava I, et al. Prevention of recurrent urinary tract infections by intravesical administration of hyaluronic acid and chondroitin sulphate: a placebo-controlled randomized trial. Eur Urol. 2011;59(4):645-651.

42. Pannek J, Pannek-Rademacher S, Wöllner J. Use of complementary and alternative medicine in persons with spinal cord injury in Switzerland: a survey study. Spinal Cord. 2015;53(7):569-572.

43. Aune A, Alraek T, LiHua H, Baerheim A. Acupuncture in the prophylaxis of recurrent lower urinary tract infection in adult women. Scand J Prim Health Care. 1998;16(1):37-39.

44. Alraek T, Baerheim A, Birch S. Acupuncture points used in the prophylaxis against recurrent uncomplicated cystitis, patterns identified and their possible relationship to physiological measurements. Chin J Integr Med. 2016;22(7):510-517.

45. Pannek J, Pannek-Rademacher S, Jus MC, Jus MS. Usefulness of classical homoeopathy for the prevention of urinary tract infections in patients with neurogenic bladder dysfunction: a case series. Indian $J$ Res Homoeopathy. 2014;8(1):31-36.

46. Jonas WB, Kaptchuck TJ, Linde K. A critical overview of homeopathy. Ann Intern Med. 2003;138(5):393-399.

47. Christensen P, Bazzocchi G, Coggrave M, et al. A randomized, controlled trial of transanal irrigation versus conservative bowel management in spinal cord-injured patients. Gastroenterology. 2006;131(3):738-747.

48. Emmanuel A, Kumar G, Christensen P, et al. Long-term costeffectiveness of transanal irrigation in patients with neurogenic bowel dysfunction. PLoS One. 2016;11(8):e0159394.

49. Viertel TM, Ritter K, Horz HP. Viruses versus bacteria - novel approaches to phage therapy as a tool against multidrug-resistant pathogens. J Antimicrob Chemother. 2014;69(9):2326-2336. 
50. Azeredo J, Sutherland IW. The use of phages for the removal of infectious biofilms. Curr Pharm Biotechnol. 2008;9(4):261-266.

51. Gu J, Liu X, Li Y, et al. A method for generation phage cocktail with great therapeutic potential. PLoS One. 2012;7(3):e31698.

52. Sybesma W, Zbinden R, Chanishvili N, et al. Bacteriophages as potential treatment for urinary tract infections. Front Microbiol. 2016;7:465.

53. Chibeu A, Lingohr EJ, Masson L, et al. Bacteriophages with the ability to degrade uropathogenic Escherichia coli biofilms. Viruses. 2012;4(4): 471-487.
54. Khawaldeh A, Morales S, Dillon B, et al. Bacteriophage therapy for refractory Pseudomonas aeruginosa urinary tract infection. $J$ Med Microbiol. 2011;60(Pt 11):1697-700.

55. Forum Urodynamicum e.V. Jahrestagung Forum Urodynamicum 2017 10. bis 11. Marz 2017 in Wiesbaden [Anniversary Forum Urodynamicum 2017; 10th to 11th March 2017 in Wiesbaden]. Available from: http://www.forum-urodynamicum.de/Jahrestagung_2017/Programm_ FU_2017.pdf. Accessed June 15, 2017.

\section{Publish your work in this journal}

Research and Reports in Urology is an international, peer-reviewed, open access journal publishing original research, reports, editorials, reviews and commentaries on all aspects of adult and pediatric urology in the clinic and laboratory including the following topics: Pathology, pathophysiology of urological disease; Investigation and treatment of

\section{Dovepress}

urological disease; Pharmacology of drugs used for the treatment of urological disease. The manuscript management system is completely online and includes a very quick and fair peer-review system, which is all easy to use. Visit http://www.dovepress.com/testimonials.php to read real quotes from published authors. 